\title{
Novel 3D "active" representations of skin biomechanics
}

\author{
Novas representações $3 D$ "ativas" da biomecânica cutânea
}

\author{
Henrique Silva ${ }^{1,2}$, Francisco FC Rego ${ }^{3,4}$, Catarina Rosado ${ }^{1}$, L Monteiro Rodrigues ${ }^{1,2}$ \\ ${ }^{1}$ Universidade Lusófona, School of Health Sc \& Technologies, CBiOS (Research Center for Biosciences and Health \\ Technologies), Campo Grande 376, 1749-024, Lisboa, Portugal \\ ${ }^{2}$ Universidade de Lisboa, Faculty of Pharmacy, Pharmacol. Sc Depart - Lisboa, Portugal \\ ${ }^{3}$ École Polytechnique Fédérale de Lausanne (EPFL), School of Engineering (STI), Automatic Control Laboratory 3 (LA3), \\ Lausanne, Switzerland \\ ${ }^{4}$ Universidade de Lisboa, Instituto Superior Técnico, Institute for Systems and Robotics (ISR), Lisboa, Portugal \\ E-mail: henrique.silva@ulusofona.pt
}

\begin{abstract}
Skin exhibits unique biomechanical properties that enable unrestricted body movements without tearing. Several devices have been used to quantify skin mechanical properties, but techniques, in general, do not concern this multidirectional capacity, only allowing measurements in a few angles. CutiScan ${ }^{\circledR}$ is a new device that quantifies skin elasticity over $360^{\circ}$. It uses a suction method to induce skin deformation and a video camera to quantify its displacement. This work aims to assess these properties through the analysis of 3D time-angle-height of displacement representations. 20 female subjects $(37.0 \pm 18.7$ years old $)$ were enrolled in this study after informed consent, grouped by age in group $1(22.0 \pm 1.3$ years old $)$, and group $2(52.0 \pm 13.7$ years old $)$. The in vivo mechanical profile of each volunteer was assessed in the forehead, forearm and in the leg. Significantly higher surface area and volume under the curve values were found in the forehead of the subjects of group 2. Significant differences were also found between the forehead and forearm and between the forehead and leg among each group. These results suggest that these 3D representations are useful in distinguishing the viscoelastic profile of differently aged subjects and of different skin sites.
\end{abstract}

Keywords: skin biomechanics; 3D representation variables; CutiScan ${ }^{\circledR}$, age differences

\section{Resumo}

A pele apresenta propriedades biomecânicas únicas que permitem a realização de movimentos corporais sem restrições sem sofrer rutura. Vários equipamentos têm sido usados para quantificar as propriedades biomecânicas, no entanto as técnicas não têm em conta esta capacidade multidirecional, permitindo apenas medições em alguns ângulos. O CutiScan ${ }^{\circledR}$ é um novo equipamento que quantifica a elasticidade cutânea ao longo de $360^{\circ}$. Utiliza um método de sucção que induz deformação cutânea e uma câmara que quantifica a sua distensão. Este trabalho tem como objetivo avaliar estas propriedades através da análise de representações 3D tempo-ângulo-altura de distensão. 20 voluntários do sexo feminino ( $37.0 \pm 18.7$ anos de idade) participaram neste estudo após consentimento informado, agrupados por idades em Grupo 1 (22.0 \pm 1.3 anos) e Grupo 2 (52.0 \pm 13.7 anos). O perfil mecânico in vivo de cada voluntária foi avaliado na fronte, antebraço e na perna. Foram encontrados valores significativamente mais elevados de área de superfície e volume sob a superfície na fronte das voluntárias do Grupo 2. Foram também encontradas diferenças significativas entre a fronte e o antebraço e entre a fronte e a perna dentro de cada grupo, Estes resultados sugerem que estas representações 3D são úteis para distinguir o perfil viscoelástico de indivíduos de diferentes idades e de diferentes zonas da pele.

Palavras-chave: biomecânica cutânea; representações 3D; CutiScan®; diferenças de idade 


\section{Introduction}

Skin exhibits both viscoelastic and anisotropic properties in vivo, mainly attributable to its specific structure and composition in terms of collagen, elastic fibrils and proteoglycans ${ }^{[1,2]}$. During the application of a force, collagen fibrils suffer viscous sliding during alignment with the force direction, while elastic fibers allow shape recovery after deformation ${ }^{[1]}$. These properties allow the skin to virtually accompany all body movements, in all directions, without breaking, and therefore to effectively protect internal organs from trauma ${ }^{[1]}$.

Several devices have been used to assess the skin's viscoelastic behavior, using different methods ${ }^{[3-7]}$. However, none of them is able to perform a successful analysis along a wide range of angles. CutiScan ${ }^{\circledR}$ (CourageKhazaka FRG) was recently released to quantify skin elasticity from a 360 angle perspective, and therefore, has the potential to solve the angle limitations. The CutiScan ${ }^{\circledR}$ probe possesses a unit that performs a suction of the skin of a certain period of time, and measures the displacement of the skin caused by that suction in terms of distance in pixels ${ }^{[8]}$. It then gives the displacement distance over time for each of the 360 degrees. The aim of this study was to use these time-distance displacement curves to construct three-dimensional (3D) representations of the in vivo biomechanical behavior, and compare them within different anatomical sites and between subjects of different ages.

\section{Materials and Methods}

20 healthy subjects (female, mean age $37.0 \pm 18.7$ years old) were selected after informed written consent. Volunteers were divided in two groups according to age (Group 1: $22.0 \pm 1.3$ years old, and Group 2: $52.0 \pm$ 13.7 years old). All procedures were conducted in full accordance with the principles of the Declaration of Helsinki and respective amendments ${ }^{[9]}$. Non-inclusion criteria included race (other than Caucasian) eventual pregnancy or lactation, any manifestation of chronic disease and/or any regular medication, presence of cutaneous signs in the experimental area, the existence of any type of topical treatment in the eight days preceding the study, and prolonged exposure to UV radiation. Additionally, some restrictions were imposed on the volunteers, including prohibition of application of topical products (such as creams, makeup and others) in the areas to be analyzed for at least 48 hours preced-

\section{Introdução}

A pele apresenta propriedades viscoelásticas e anisotrópicas in vivo, principalmente atribuíveis à sua estrutura específica e composição em termos de colagénio, fibrilhas elásticas e proteoglicanos ${ }^{[1,2]}$. Durante a aplicação de uma força, as fibrilhas de colagénio sofrem um deslizamento viscoso durante o alinhamento com a direção da força, enquanto que as fibrilhas elásticas permitem a recuperação da forma após a deformação ${ }^{[1]}$. Estas propriedades permitem à pele acompanhar virtualmente todos os movimentos corporais, em todas as direções, sem quebrar e, como tal, proteger efetivamente os órgãos internos do trauma ${ }^{[1]}$. Vários equipamentos têm sido utilizados para avaliar o comportamento viscoelástico da pele, utilizando diferentes métodos ${ }^{[3-7]}$. No entanto, nenhum deles é capaz de realizar uma análise satisfatória ao longo de um intervalo grande de ângulos. O CutiScan ${ }^{\circledR}$ (Courage-Khazaka FRG) foi lançado recentemente para a quantificação da elasticidade da pele numa perspetiva de 360 ângulos e, como tal, com o potencial de eliminar estas limitações angulares. A sonda do CutiScan ${ }^{\circledR}$ possui uma unidade que leva a cabo a sucção da pele de uma determinada duração, e mede a distensão da pele causada por essa sucção em termos de distância em píxeis ${ }^{[8]}$. Devolve, então, a distância de distensão ao longo do tempo para cada um dos 360 graus. O objetivo deste estudo foi o de utilizar estas curvas de tempo-distância de distensão para construir representações tridimensionais (3D) do perfil biomecânico in vivo, e compará-las, dentro de diferentes regiões anatómicas e entre indivíduos de diferentes idades.

\section{Materiais e Métodos}

20 voluntários saudáveis (sexo feminino, idade média $37.0 \pm 18.7$ anos) foram selecionadas após consentimento informado. As voluntárias foram divididas em dois grupos de acordo com a idade (Grupo 1: $22.0 \pm 1.3$ anos, e Grupo 2: $37.0 \pm 18.7$ anos). Todos os procedimentos foram conduzidos de acordo com os princípios da Declaração de Helsínquia e emendas respetivas ${ }^{[9]}$. Critérios de não-inclusão incluíram a raça (outras que não a caucasiana), eventual gravidez ou aleitamento, qualquer manifestação de doença crónica e/ou qualquer medicação regular, presença de sinais cutâneos na área experimental, a existência de qualquer tipo de tratamento tópico nos oito dias que antecederam o estudo e exposição prolongada a radiação UV. Foram também impostas algumas restrições às voluntárias, incluindo a proibição de aplicação de produtos tópicos (como cremes, maquilhagem e outros) nas áreas a serem anali- 
ing the measurements. All measurements were carried out under constant environmental conditions $\left(20-22^{\circ} \mathrm{C}\right)$ and $40-60 \%$ relative humidity) and according to previously published methods and recommendations ${ }^{[10]}$. Three different anatomical sites were chosen for measurement purposes - forehead, forearm and lower leg. A CutiScan ${ }^{\circledR}$ prototype (Courage-Khazaka FRG) was used to quantify the in vivo skin's biomechanics. This suction method provides a time/strain measurement mode and a suction cycle with a constant pressure of 400 mbar for about 3 seconds, followed by a relaxation period of about 3 seconds. The analysis software calculates the following parameters ${ }^{[8]}$ :

- V1: maximum displacement during suction time;

- V2: The returning rate during the relaxation time;

- V3: the ratio of V2/V1.

The first 2 seconds of the suction phase were then used to construct two different 3D representations of the elasticity profile - a polar representation and a nonpolar representation. The nonpolar representation is a simple 3D plotting of displacement over time over angle. The polar representation is a plotting of displacement over the 'time times sine(angle)' over 'time time cos(angle)'. The 'surface area (SA)' and the 'volume under the surface (VUS)' of these representations were then calculated using Matlab-based algorithms. Values were compared with the Mann-Whitney test for independent samples (SPSS 21.0, Microsoft) and a significance level of $95 \%$ was adopted $(p<0.05)$.

\section{Results and Discussion}

Table 1 summarizes the SA and VUS calculations done for the $3 \mathrm{D}$ polar representation. Tendencies were similar for both group's 3D representations when comparing young with older subjects, with older subjects showing lower SA and VUS values for both the forearm and leg. No statistically significant differences could be found. Nevertheless, significantly higher SA and VUS where found in the forehead of older subjects. Similar results were found for the nonpolar representation, as shown in Table 2. Once again SA and VUS values were lower on the forearm and leg in older subjects, but without statistical significance, whereas in the forehead both values sadas no mínimo nas 48 horas que precederam as medições. Todas as medições foram levadas a cabo em condições ambientais constantes $\left(20-22^{\circ} \mathrm{C}\right)$ e humidade relativa de $40-60 \%$ e de acordo com métodos e recomendações previamente publicados ${ }^{[10]}$.

Foram escolhidas três zonas anatómicas diferentes para os efeitos de medição - fronte, antebraço e perna. Um protótipo do CutiScan ${ }^{\circledR}$ (Courage-Khazaka FRG) foi usado para quantificar a biomecânica cutânea in vivo. Este método de sucção fornece um modo de medição de tempo/tensão e um ciclo de sucção com uma pressão constante de 400 mbar durante aproximadamente 3 segundos, seguido de um período de relaxamento de aproximadamente 3 segundos. O software de análise calcula os seguintes parâmetros ${ }^{[8]}$ :

- V1: distensão máxima durante o período de sucção;

- V2: a taxa de recuperação durante o período de relaxamento;

- V3: o rácio V2/V1.

Os primeiros 2 segundos da fase de sucção foram então usados para construir duas diferentes representações 3D do perfil de elasticidade - uma representação polar e uma representação não-polar. A representação não- polar é uma simples representação 3D da distensão ao longo do tempo e do ângulo. A representação polar é uma representação da distensão ao longo do 'tempo $\times$ seno(ângulo)' e do 'tempo $\times$ cos(ângulo)'. A 'área de superfície (AS)' e o 'volume sob a superfície (VSS)' destas representações foram calculadas utilizando algoritmos com base em Matlab. Os valores foram comparados pelo teste de Mann-Whitney para amostras independentes (SPSS 21.0, Microsoft) tendo sido adotado um nível de significância de 95\% ( $\mathrm{p}<0.05)$.

\section{Resultados e Discussão}

A Tabela 1 resume os cálculos de AS e VSS para as representaçes polares em 3D. As tendências foram semelhantes para as representações 3D de ambos os grupos comparando sujeitos jovens com sujeitos com mais idade, com os sujeitos com mais idade apresentando valores AS e VSS mais baixos para o antebraço e para a perna. Não foram encontradas diferenças estatisticamente relevantes. No entanto, foram registados valores significativamente superiores de AS e VSS na fronte dos sujeitos com mais idade. Foram registados resultados semelhantes para a representação não-polar, como se observa na Tabela 2. Mais uma vez, os valores de 
Table 1/ Tabela 1 - Mean and standard deviation (sd) of the surface area (SA) and volume under the surface (VUS) for the polar 3D representation $(* \mathrm{p}<0.05)$

Média e desvio-padrão (dp) da área de superfície (AS) e do volume sob a superfície (VSS) para a representação $3 \mathrm{D}$ polar $(* \mathrm{p}<0.05)$.

\begin{tabular}{|c|c|c|c|c|c|c|c|}
\hline \multirow{2}{*}{\multicolumn{2}{|c|}{$\begin{array}{c}\text { Anatomical site / Zona } \\
\text { anatómica }\end{array}$}} & \multicolumn{3}{|c|}{$\mathbf{S A} / \mathbf{A S}$} & \multicolumn{3}{|c|}{ VUS / VSS } \\
\hline & & \multirow{2}{*}{$\begin{array}{c}\begin{array}{c}\text { Group 1 } \\
\text { / Grupo } \\
\mathbf{1}\end{array} \\
293.33 \\
\end{array}$} & \multirow{2}{*}{$\begin{array}{c}\text { Group 2 / } \\
\text { Grupo 2 } \\
226.04 \\
\end{array}$} & \multirow{3}{*}{$\begin{array}{c}\begin{array}{c}\text { p value / } \\
\text { valor p }\end{array} \\
0.278\end{array}$} & \multirow{3}{*}{$\begin{array}{c}\begin{array}{c}\text { Group 1 / } \\
\text { Grupo 1 }\end{array} \\
1112.42 \\
308.47\end{array}$} & \multirow{3}{*}{$\begin{array}{c}\begin{array}{c}\text { Group 2 / } \\
\text { Grupo 2 }\end{array} \\
919.15 \\
357.35\end{array}$} & \multirow{2}{*}{$\begin{array}{c}\begin{array}{c}\text { p value / } \\
\text { valor } \mathbf{p}\end{array} \\
0.356\end{array}$} \\
\hline Forearm / & mean / média & & & & & & \\
\hline Antebraço & sd /dp & 90.05 & 109.50 & & & & 0.356 \\
\hline \multirow{2}{*}{$\begin{array}{l}\text { Leg / } \\
\text { Perna }\end{array}$} & mean / média & 232.58 & 176.09 & \multirow{2}{*}{0.315} & 947.05 & 817.69 & \multirow{2}{*}{0.740} \\
\hline & sd /dp & 81.69 & 77.75 & & 262.40 & 380.67 & \\
\hline \multirow{2}{*}{$\begin{array}{l}\text { Forehead / } \\
\text { Fronte }\end{array}$} & mean / média & 278.90 & 603.71 & \multirow{2}{*}{$0.024 *$} & 1256.11 & 2074.13 & \multirow{2}{*}{$0.024^{*}$} \\
\hline & sd /dp & 150.63 & 493.74 & & 383.65 & 1103.78 & \\
\hline
\end{tabular}

Table 2/ Tabela 2 - Mean \pm standard deviation (sd) of the surface area (SA) and the volume under the surface (VUS) for the nonpolar 3D representation $(* \mathrm{p}<0.05)$

Média e desvio-padrão (dp) da área de superfície (AS) e do volume sob a superfície (VSS) para a representação 3D não-polar $(* \mathrm{p}<0.05)$.

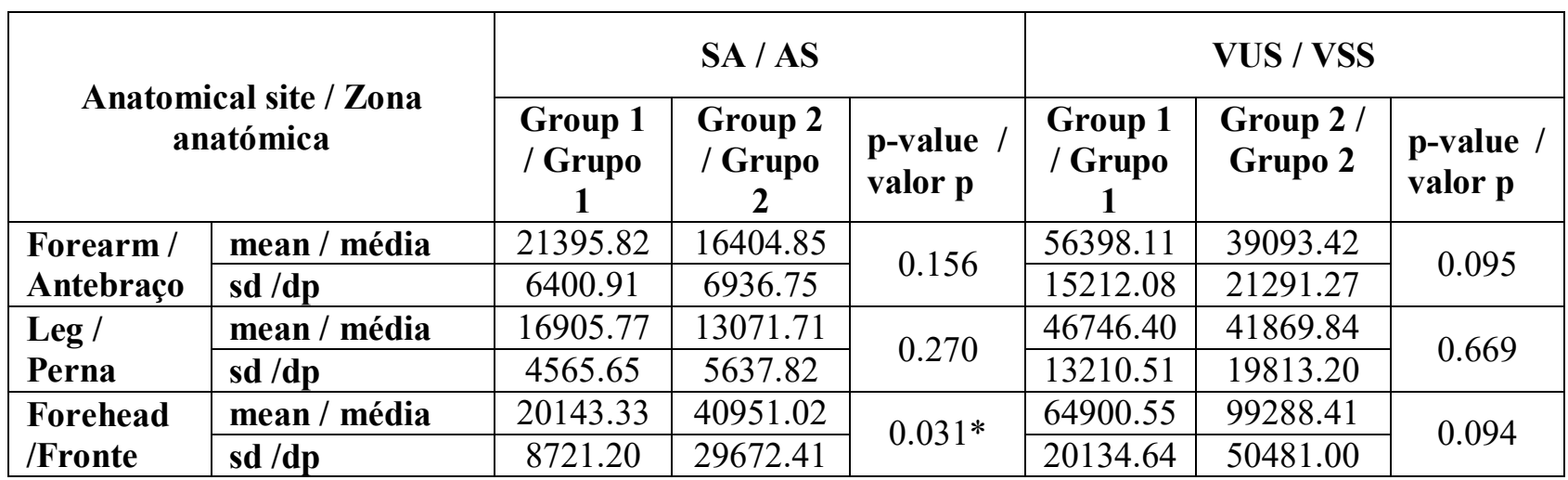


were higher on older subjects, but only significantly for SA.

Calculations resulting from both $3 \mathrm{D}$ representations are consistent regarding the differences of values between groups. Older subjects show less pronounced skin displacement on the forearm and leg, but higher displacement for the forehead. When comparing different anatomical regions, more pronounced differences were found between the forehead and limbs, as shown in Table 3. No significant differences were found for SA and VUS when comparing the forearm with the leg of both young and older subjects. When comparing the results obtained in the forearm with those of the forehead of younger subjects, no significant differences were found, but this was not the case for the older patients $(\mathrm{p}=0.004$ for SA and $\mathrm{p}<0.001$ for VUS). Again, comparing results from the forehead with the leg obtained in group 1, only VUS was found to be significantly different, while for group 2, both SA and VUS were significantly different. Skin is well known to undergo significant changes in its biomechanical behavior as a function of age, but change is also determined by other extrinsic (UV radiation, environmental, disease) factors ${ }^{[11]}$. Several papers have been published describing the effect of ageing on skin biomechanics, specially using suction methods and
AS e VSS foram inferiores no antebraço e na perna das voluntárias mais velhas, mas sem relevância estatística, enquanto que, na fronte, ambos os valores foram mais elevados nas voluntárias mais velhas, mas apenas significativamente para os valores de AS.

Os cálculos resultantes de ambas as representações 3D são consistentes em relação às diferenças dos valores entre os grupos. As voluntárias mais velhas apresentam uma distensão de pele menos pronunciada no antebraço e na perna, mas uma distensão mais elevada na fronte. Comparando diferentes regiões anatómicas, foram registadas diferenças mais pronunciadas entre a fronte $\mathrm{e}$ os membros, como está representado na Tabela 3. Não foram encontradas diferenças significativas de valores de AS e VSS comparando o antebraço com a perna, de tanto as voluntárias jovens como as mais velhas. Comparando os resultados obtidos no antebraço com os da fronte das voluntárias mais jovens, não foram encontradas diferenças significativas, mas este não foi o caso para as voluntárias mais velhas $(\mathrm{p}=0.004 \mathrm{em}$ AS e $\mathrm{p}<0.001 \mathrm{em}$ VSS). Mais uma vez, comparando os resultados da fronte com a perna obtidos no Grupo 1, apenas os valores de VSS foram significativamente diferentes, enquanto que para o Grupo 2, tanto os valores de AS como de VSS foram significativamente diferentes.

Table 3/ Tabela 3 - Comparison p values from SA versus VUS for different anatomical sites and different groups, for both $3 \mathrm{D}$ representations $(* \mathrm{p}<0.05)$.

Valores de p para a comparação entre AS e VSS entre as diferentes zonas anatómicas para ambas as representações $3 \mathrm{D}(* \mathrm{p}<0,05)$.

\begin{tabular}{|l|l|c|c|c|c|c|c|}
\cline { 3 - 8 } \multicolumn{2}{c|}{} & \multicolumn{2}{c|}{$\begin{array}{c}\text { Forearm vs Leg } \\
\text { Antebraço vs Perna }\end{array}$} & \multicolumn{2}{c|}{$\begin{array}{c}\text { Forearm vs Forehead } \\
\text { Antebraço vs Fronte }\end{array}$} & \multicolumn{2}{c|}{$\begin{array}{c}\text { Leg vs Forehead } \\
\text { Perna vs Fronte }\end{array}$} \\
\cline { 3 - 8 } & & $\begin{array}{c}\text { Group 1 / } \\
\text { Grupo 1 }\end{array}$ & $\begin{array}{c}\text { Group 2 } \\
\text { / Grupo } \\
\mathbf{2}\end{array}$ & $\begin{array}{c}\text { Group 1 / } \\
\text { Grupo 1 }\end{array}$ & $\begin{array}{c}\text { Group 2 / } \\
\text { Grupo 2 }\end{array}$ & $\begin{array}{c}\text { Group 1 } \\
\text { / Grupo } \\
\mathbf{1}\end{array}$ & $\begin{array}{c}\text { Group 2 / } \\
\text { Grupo 2 }\end{array}$ \\
\hline \multirow{2}{*}{ Polar / Polar } & SA / AS & 0.165 & 0.408 & 0.400 & $0.008^{*}$ & 0.604 & $0.001^{*}$ \\
\cline { 3 - 9 } & VUS / VSS & 0.165 & 0.47 & 0.447 & $<0.001^{*}$ & $0.043^{*}$ & $<0.001^{*}$ \\
\hline \multirow{2}{*}{$\begin{array}{l}\text { Non-polar / Não } \\
\text { polar }\end{array}$} & SA / AS & 0.165 & 0.252 & 0.72 & $0.004^{*}$ & 0.278 & $0.001^{*}$ \\
\cline { 2 - 9 } & VUS / VSS & 0.123 & 0.758 & 0.356 & $<0.001^{*}$ & $0.035^{*}$ & $0.002^{*}$ \\
\hline
\end{tabular}


related quantitative descriptors ${ }^{[12,13]}$. But a true 3D representation of the "active" phenomena involved after suction, described in terms of time, angle, and height displacement, has not been attempted previously (the term "active" is used by opposition to the 3D representation of the skin surface obtained by profilometry). In fact these polar and non-polar representations do seem to illustrate the variations of V1, V2 and V3 ${ }^{[8]}$ detected by the CutiScan ${ }^{\circledR}$ and reveal the expected evolution resulting from these parameters. A clear change in the representation patterns is consistently found in group 2, compared to group 1. Patients were all healthy and Caucasian, hence especially prone to the pro-age-
É bem conhecido que a pele sofre mudanças significativas no seu compertamento biomecânico em função da idade, mas estas mudanças são também determinadas por outros factores extrínsecos (radiação UV, ambientais, doenças) ${ }^{[11]}$. Foram publicados numerosos artigos nos quais é descrito o efeito da idade na biomecânica da pele, especialmente recorrendo ao uso de métodos de sucção e representações quantitativas relacionadas ${ }^{[12,13]}$. No entanto, não foi ainda realizada uma verdadeira representação 3D dos fenómenos "ativos" envolvidos, depois da sucção, descritos em termos de tempo, ângulo, e distenção vertical (o termo "ativo" é usado em contraste com a representação $3 \mathrm{D}$ da superfície da

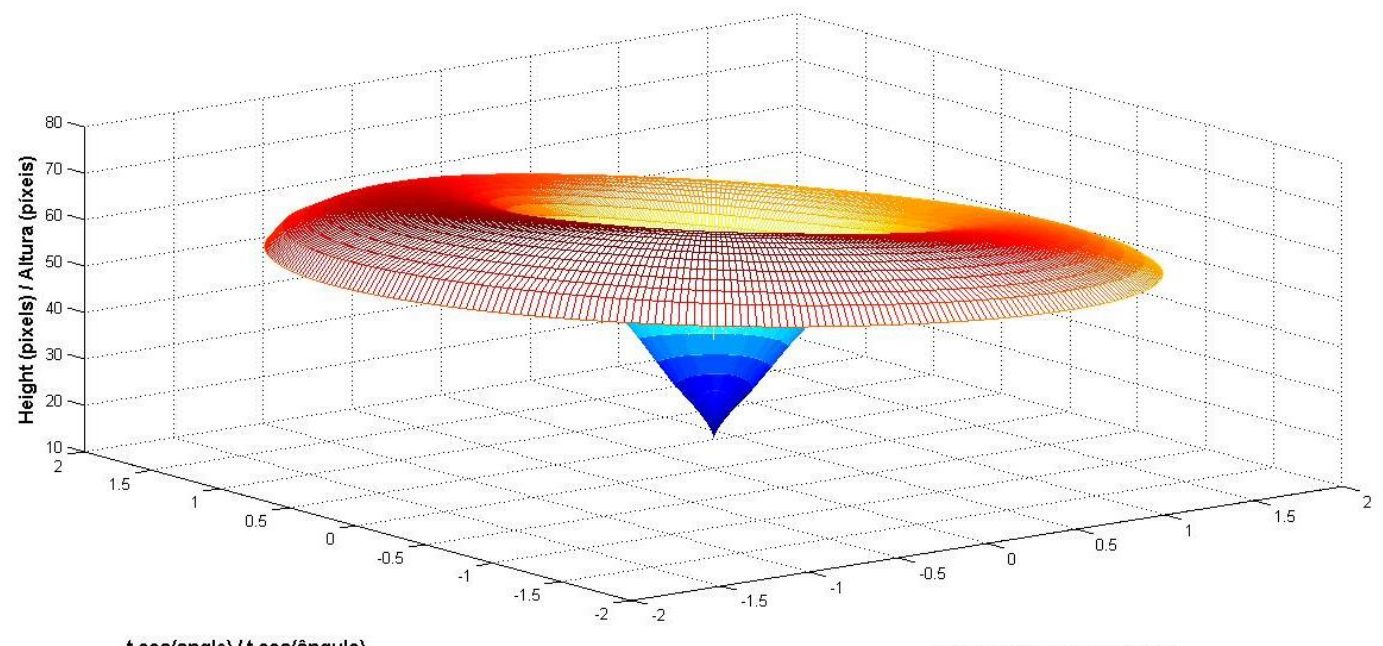

t.cos(angle)/t.cos(ângulo)

t.sin(angle)/ t.seno(ângulo)

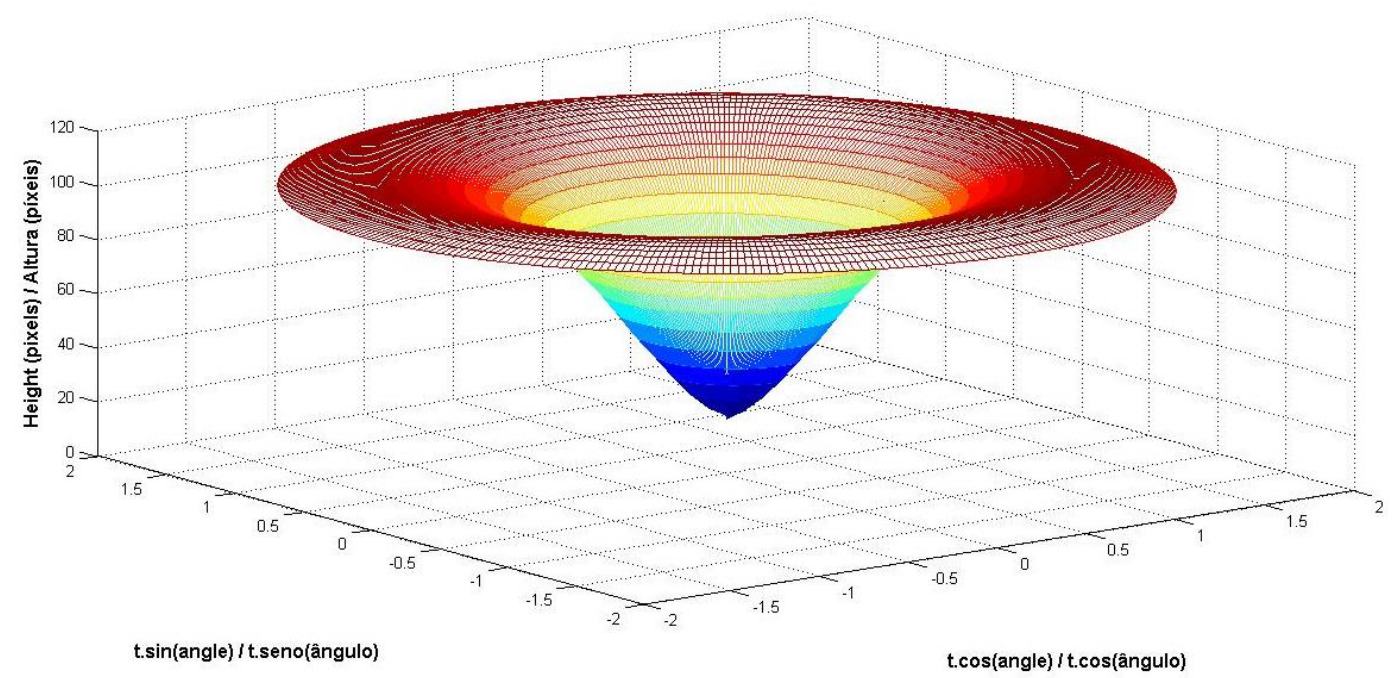

Figure 1/ Figura 1 - Average 3D polar representations of groups 1 (top) and 2 (bottom). The group 1 representation is both shorter and tighter at its apex in comparison with group 2, which means a less pronounced, albeit faster, skin distension over all angles upon suction, respectively/ Representações 3D polares médias dos grupos 1 (em cima) e 2 (em baixo). A representação do Grupo 1 é mais curta e mais apertada no ápice em comparação com a do Grupo 2, o que significa uma distensão menos pronunciada mas mais rápida ao longo de todos os ângulos durante a sucção, respectivamente. 

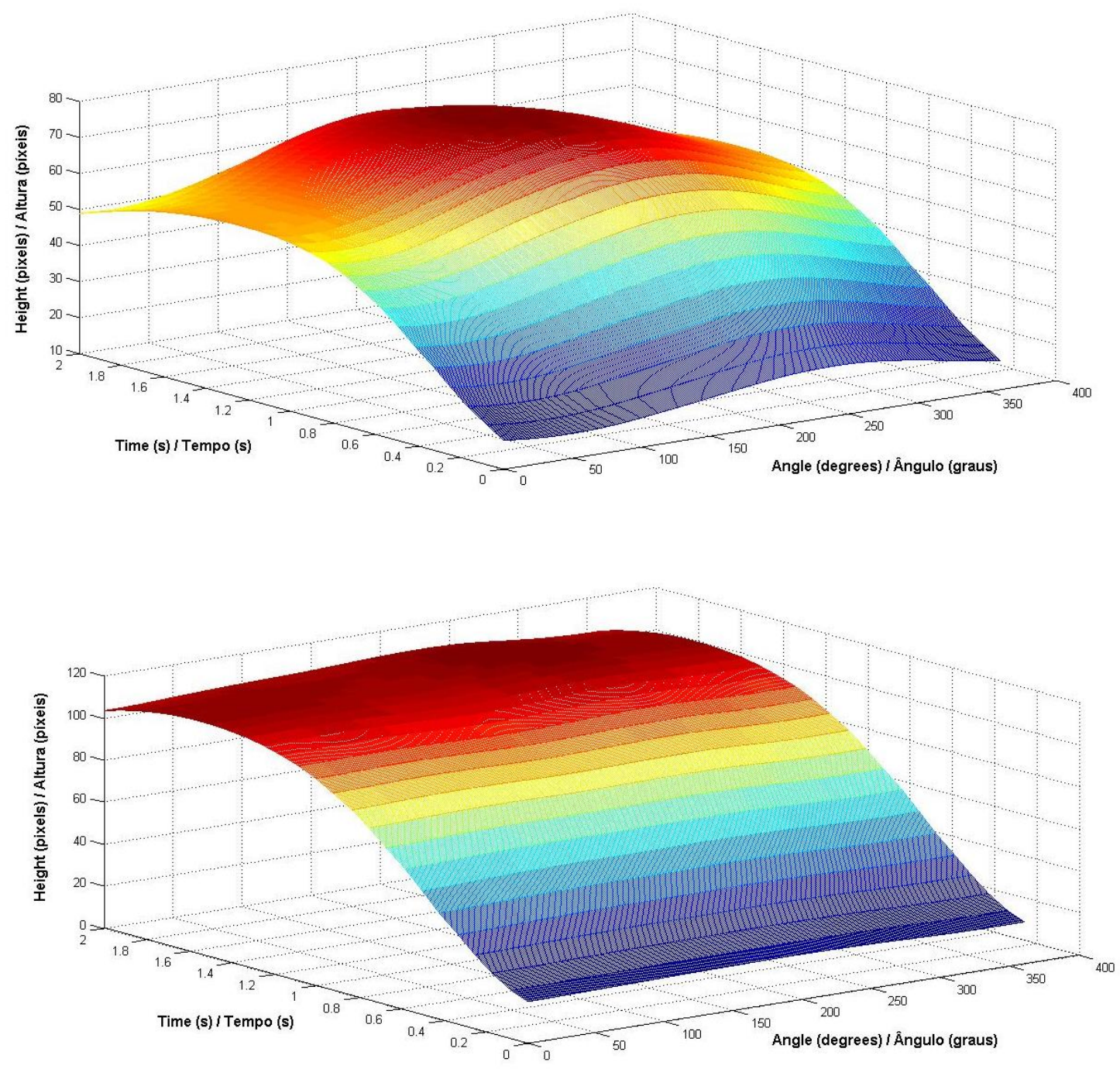

Figure 2/ Figura 2 - Average 3D nonpolar representations of groups 1 (top) and 2 (bottom). Group 1 representation is shorter in all angles comparing with group 2, which means a less pronounced skin distension upon suction. Representation for both groups shows a maximal distension approximately between 100 and $200^{\circ}$ and time 1 to 2 seconds, while the maximal distension for group 2 is more evenly distributed over all angles. This means that a preferential orientation of maximal skin distension is found for group 1 but not for group 2.

Representações 3D não-polares médias para os grupos 1 (em cima) e 2 (em baixo). A representação do Grupo 1 é mais curta ao longo de todos os ângulos, em comparação com a do Grupo 2, o que significa uma menor distensão da pele durante a sucção. As representações para ambos grupos mostram uma distensão máxima aproximadamente entre os 100 e $200^{\circ}$ e entre os 1 e 2 s, enquanto a distensão máxima para o Grupo 2 está mais igualmente distribuída ao longo de todos os ângulos. Tal significa existir uma orientação preferencial de distensão máxima para o Grupo 1 mas não para o Grupo 2.

ing effects of UV radiation ${ }^{[12]}$. Differences, more pronounced in the forehead, likely reflect this special condition of facial skin, simultaneously the thinnest and the most exposed in the entire body (the protective effect of clothing preserves all other areas, including the forearm and the leg). pele obtida por medição de perfil). De facto, estas representações polares e não-polares aparentam ilustrar as variações de V1, V2 e V3 ${ }^{[8]}$ detectadas pelo CutiScan ${ }^{\circledR}$ e revelam a evolução esperada resultante destes argumentos. Foi registada consistentemente uma alteração clara nos padrões de representação no Grupo 2, em comparação com o Grupo 1. Todas as voluntárias eram saudáveis e de raça causasiana, e portanto especialmente suscetíveis aos efeitos de envelhecimento da radia- 


\section{Conclusion}

These results, although preliminary, do suggest that these 3D representations and respective quantitative variables are useful to visually identify the skin elasticity profile between subjects of different ages as well as between different anatomical sites. Further studies should be performed in order to confirm the potential of this analysis procedure, in particular in clinical terms.

\section{Acknowledgements}

Francisco F.C. Rego benefited from the grant SFRH/ BD/51929/2012 of FCT, Portugal.

The authors also express their thanks for the experimental support from Filipa Antunes, Raquel Barbosa, and Raquel Fernando.

\section{Conflict of Interests}

The authors declare that there is no financial or personal relationship that can be understood as presenting a potential conflict of interest. ção UV ${ }^{[12]}$. As diferenças, mais pronunciadas na fronte, refletem provavelmente esta condição especial da pele facial, simultaneamente a mais fina e mais exposta do corpo inteiro (o efeito protetor do vestuário preserva todas as outras zonas, incluindo o antebraço e a perna).

\section{Conclusão}

Os resultados obtidos, apesar de preliminares, sugerem que estas representações $3 \mathrm{D}$ e as respectivas variáveis quantitativas são úteis para identificar visualmente o perfil de elasticidade da pele entre sujeitos de idades diferentes assim como entre diferentes zonas anatómicas. Deverão ser realizados estudos mais aprofundados para confirmar o potencial deste procedimento de análise, em particular em termos clínicos.

\section{Agradecimentos}

Francisco F.C. Rego beneficiou da bolsa SFRH/ BD/51929/2012 da FCT, Portugal.

Os autores gostariam de expresser os seus agradecimentos a Filipa Antunes, Raquel Barbosa, e Raquel Fernando pelo seu apoio experimental.

\section{Conflito de Interesses}

Os autores declaram que não têm relações financeiras ou pessoais que possam ser entendidas como apresentando um conflito potencial de interesses. 


\section{References/ Referências}

1. Silver FH, Freeman JW, DeVore D. Viscoelastic properties of human skin and processed dermis. Skin Res Technol 2001;7(1):18-23.

2. Khatyr F, Imberdis C, Vescovo P, Varchon D, Lagarde JM. Model of the viscoelastic behaviour of skin in vivo and study of anisotropy. Skin Res Technol 2004;10(2):96-103.

3. Guan Y, Lu M, Shen Z, Wan M. Optical tracking of local surface wave for skin viscoelasticity. Med Eng Phys. 2014;36(6):70814.

4. Li C, Guan G, Reif R, Huang Z, Wang RK Determining elastic properties of skin by measuring surface waves from an impulse mechanical stimulus using phase-sensitive optical coherence tomography. J R Soc Interface 2012; 9(70):831-41

5. Palma L, Marques LT, Bujan J, Rodrigues LM. Dietary water affects human skin hydration and biomechanics. Clin Cosmet Investig Dermatol 2015; 8:413-421.

6. Rodrigues L. EEMCO guidance to the in vivo assessment of tensile functional properties of the skin. Part 2: Instrumentation and test modes. Skin Pharmacol Applied Skin Physiol 2001; 14(1): 52-67.
7. Piérard GE. EEMCO guidance to the in vivo assessment of tensile functional properties of the skin. Part 1: Relevance to the structures and ageing of the skin and subcutaneous tissues. Skin Pharmacology and Applied Skin Physiology 1999; 12(6): 352-362.

8. Courage+Khazaka. Information and Operating Instruction for the CutiScan ${ }^{\circledR}$ CS 100 . Köln, Germany. 2014 Oct.

9. World Medical Association, 2013. World Medical Association Declaration of Helsinki: ethical principles for medical research involving human subjects. JAMA310, 21912194.

10. Clarys P, Barel A. Quantitative evaluation of skin surface lipids. Clinics in Dermatology 1995; 13(4): 307-21.

11. Pan L, Zan L, Foster FS. Ultrasonic and viscoelastic properties of skin under transverse mechanical stress in vitro. Ultrasound Med Biol 1998; 4(7):995-1007.
12. Choi J W, Kwon SH, Huh CH, Park KC, Youn SW. The influences of skin viscoelasticity, hydration level and aging on the formation of wrinkles: a comprehensive and objective approach. Skin Res Technol 2013;,19(1): 349-55.

13. Krueger N, Luebberding S, Oltmer $M$, Streker M, Kerscher M. Age-related changes in skin mechanical properties: a quantitative evaluation of 120 female subjects. Skin Res Technol 2011; 17(2): 141-148

14. Imokawa G. Mechanism of UVB-induced wrinkling of the skin: paracrine cytokine linkage between keratinocytes and fibroblasts leading to the stimulation of elastase. The journal of investigative dermatology. 2009; 14(1):36-43 